\title{
An automatised hydrogen orientation procedure for neutron protein crystallography
}

\author{
J. Bergmann ${ }^{1}$, E. Oksanen ${ }^{2}$, U. Ryde ${ }^{1}$ \\ ${ }^{1}$ Division of Theoretical Chemistry, Lund University, Chemical Centre, P.O. Box 124, SE-221 00 Lund, Sweden, \\ ${ }^{2}$ European Spallation Source ESS ERIC, Lund, Sweden
}

Justin.Bergmann@teokem.lu.se

Single-crystal neutron scattering experiments have the advantage compared to X-ray experiments that it is possible to get positions for hydrogen atoms - typically replaced by deuterons in neutron protein crystallography. The hydrogens are important because they constitute approximately half of the atoms in a protein and determine the directionality of hydrogen bonds, which are key to the structure and function [1]. Therefore, neutron crystallographic experiments give important additional information to the model. However, adding all hydrogens by hand to the model is a tedious and error-prone work and most software add hydrogens at positions suggested by a statistical analysis of neutron structures and not based on the measured data. Moreover, it is important to decide for each hydrogen atom whether its position is supported by the experimental data or not.

To solve these problems, we developed an automatised procedure that places all hydrogen atoms of a protein based on local integration of the neutron $2 m F_{\mathrm{o}}-D F_{\mathrm{c}}$ map. For each putative hydrogen atom, we search for the highest integrated value of the nuclear scattering length density within a sphere of the covalent radius of hydrogen. For some hydrogens, the position is dictated by the positions of the surrounding heavy atoms. However, many hydrogens can be anywhere on a circle (e.g. $\mathrm{OH}, \mathrm{SH}$ and $\mathrm{NH}_{3}$ groups) and we search all possible positions systematically for the highest integrated density. Likewise, we consider possible flips of Asn and Gln residues, we consider six possible states of His residues, and we consider alternative protonation states of Asp, Glu, Lys, Tyr and Cys residues. The method is calibrated to available neutron structures and the number of favourable hydrogen bonds are evaluated.

[1] Engler, N., Ostermann, A., Niimura, N., \& Parak, F. G. (2003). Hydrogen atoms in proteins: positions and dynamics, Proc. Natl. Acad. Sci. U.S.A., 100(18), 10243-10248.

Keywords: Neutron crystallography, Model building, Hydrogen position, Automatising 